\title{
Polarised correlators at finite temperature
}

\section{G. Aarts, C.R. Allton*, S.J. Hands}

Department of Physics, Swansea University, Swansea, U.K.

\section{J. Foley}

Carnegie Mellon University, Pittsburgh, USA

\section{S. Kim}

Sejong University, Seoul, Korea

QCD undergoes a deconfining transition at high temperature to a "quark-gluon plasma" phase where hadrons may become unbound. In this work, meson correlation functions at non-zero momentum are studied both in the confined and plasma phases using the Maximum Entropy Method. In particular, both the longitudinal and transverse modes of the vector correlation functions are considered. Only in the case of light quarks in the plasma phase, we find that both longitudinal and transverse spectral functions have a non-zero intercept at zero energy.

XXVIIIth International Symposium on Lattice Field Theory

June 14-19, 2010

Villasimius, Sardegna, Italia

${ }^{*}$ Speaker. 


\section{Introduction}

There has been considerable interest in the quark-gluon plasma phase of QCD over recent years. On the experimental side, experiments at RHIC have led to an increased understanding of this phase of matter. This has been matched by a similarly intense theoretical understanding using e.g. hydrodynamic models and lattice simulations. Despite this considerable work, there remains a great deal of uncertainty over many properties of the quark-gluon plasma. In particular, the Particle Data Book does not contain a single entry for this phase [1].

This work aims to extend our knowledge of the physics of QCD in the plasma phase by studying the properties of mesonic spectral functions, $\rho(\omega)$, both below and above the deconfining temperature, $T_{c}$. The zero energy limit, $\omega \rightarrow 0$, of spectral functions gives information on hydrodynamic structure and transport coefficients; we will be particularly interested in the conductivity, $\sigma$, and the related diffusivity, $D=\sigma / \Xi$, where $\Xi$ is the charge susceptibility. These can be obtained from vector spectral functions. In this paper, we outline an extension of our earlier work [2] to the case of non-zero momentum [3]. This allows us to study the longitudinal and transverse components in the vector channel which can, in principle, unlock interesting information about transport and hydrodynamics [4]. For more details on transport coefficients and lattice QCD, we refer to the reviews $[5,6]$.

\section{Lattice and Fitting Details}

We begin by defining the usual spectral function in terms of the Euclidean two-point function, $G(t, \vec{p})$,

$$
G(t, \vec{p})=\int \frac{d \omega}{2 \pi} \rho(\omega, \vec{p}) K(t, \omega),
$$

where $\vec{p}$ denotes the momentum. The kernel is given by

$$
K(t, \omega)=\frac{\cosh [\omega(t-1 /(2 T))]}{\sinh [\omega /(2 T)]},
$$

where $T=1 /\left(a N_{t}\right)$. We note that the extraction of a spectral density from a lattice correlator is an ill-posed problem, since the correlator, $G(t)$, is known at only $\mathscr{O}(10)$ time points, whereas the spectral function, $\rho(\omega)$, is, in principle, a continuous function. The usual solution of this problem implements the Bayesian analysis technique of the Maximum Entropy Method (MEM) [7].

At finite temperature the kernel is singular and independent of euclidean time in the limit that $\omega \rightarrow 0[8]:$

$$
\lim _{\omega \rightarrow 0} K(\omega, t)=\frac{2 T}{\omega}+\mathscr{O}(\omega) .
$$

In Ref. [2] we uncovered that this singularity affects the reliability of the MEM procedure near $\omega=0$. Fortunately it can be trivially corrected by defining a rescaled kernel and spectral function,

$$
\bar{K}(\omega, t)=\frac{\omega}{2 T} K(\omega, t), \quad \bar{\rho}(\omega)=\frac{2 T}{\omega} \rho(\omega),
$$

and performing MEM on

$$
G(t)=\int \frac{d \omega}{2 \pi} \bar{\rho}(\omega) \bar{K}(\omega, t)
$$




\begin{tabular}{lccc}
\hline & & Cold & Hot \\
& & & \\
\hline Spatial Volume & $N_{s}^{3} \times N_{t}$ & $48^{3} \times 24$ & $64^{3} \times 24$ \\
Lattice spacings & $a^{-1}$ & $\sim 4 \mathrm{GeV}$ & $\sim 10 \mathrm{GeV}$ \\
$T$ & $1 /\left(a N_{t}\right)$ & $T \sim 160 \mathrm{MeV} \sim 0.62 T_{c}$ & $T \sim 420 \mathrm{MeV} \sim 1.5 T_{c}$ \\
Statistics & $N_{c f g}$ & $\sim 100$ & $\sim 100$ \\
Quark Masses & $m a$ & $0.01 \& 0.05$ & $0.01 \& 0.05$ \\
& & & \\
\hline
\end{tabular}

Table 1: Lattice parameters used in the simulation. Estimates for the lattice spacing and temperature are taken from Ref. [10].

We demonstrated that this redefinition removes the unphysical behaviour of the spectral functions constructed with MEM near $\omega=0$, present when the traditional kernel is employed [2].

In the MEM procedure, we used the default model

$$
\bar{m}(\omega)=m_{0}(1+a \omega)
$$

with $m_{0}$ determined from a fit of $\int d \omega \bar{m}(\omega) \bar{K}(\omega)$ to the data. This definition of $\bar{m}(\omega)$ matches the expected perturbative behaviour $\rho(\omega) \sim \omega \bar{\rho}(\omega) \sim \omega^{2}$ as $\omega \rightarrow \infty$ and also allows a non-zero intercept in $\rho / \omega \sim \bar{\rho}$ (corresponding to a transport peak) as $\omega \rightarrow 0$. For a study of lattice spectral functions at nonzero momentum in the infinite temperature limit, see Ref. [9].

The lattice action and parameters used are identical to those in Ref. [2], i.e. a simple Wilson plaquette action with quenched, staggered fermions. The lattice parameters used are displayed in Table 1. In Ref. [2] we determined the electrical conductivity, $\sigma / T=0.4 \pm 0.1$, a result which has since been confirmed [11]. We extend the work in Ref. [2] further by using twisted boundary conditions with the same choices of twists as in Ref. [12]. This enables us to access a large range of momenta which are listed in Table 2. Preliminary results can be found in Ref. [3].

Since we use staggered correlators which have contributions from both parity partners,

$$
G(t)=\int \frac{d \omega}{2 \pi} K(t, \omega)\left[\rho(\omega)-(-1)^{t / a} \bar{\rho}(\omega)\right],
$$

we are forced to apply MEM to even and odd times separately, and then reconstruct the physical spectral function from

$$
\rho^{\text {phys }}=\frac{1}{2}\left(\rho^{\text {even }}+\rho^{\text {odd }}\right) \text {. }
$$

\section{Longitudinal and Transverse Correlators}

In Fig. 1 we show the vector correlators for both the longitudinal and transverse modes, $G_{\mathrm{L}, \mathrm{T}}$, for momenta $p L=2, \pi, 2 \pi, 3 \pi$ as a function of euclidean time. We display these as a ratio with the average correlation, $G_{\mathrm{Ave}}=\left(G_{\mathrm{L}}+2 G_{\mathrm{T}}\right) / 3$. Note that we use local (rather than conserved) currents 


\begin{tabular}{cccc}
\hline$\vec{p} L$ & $|p| L$ & Longitudinal & Transverse \\
& & & \\
$(0,0,0)$ & 0 & - & - \\
$(2,0,0)$ & 2 & $V_{1}$ & $V_{2} \& V_{3}$ \\
$(0, \pm \pi, 0)$ & $\pi$ & $V_{2}$ & $V_{1} \& V_{3}$ \\
$(-2, \pm \pi, 0)$ & $\sqrt{4+\pi^{2}} \sim 3.72$ & - & $V_{3}$ \\
$(0, \pm 2 \pi, 0)$ & $2 \pi \sim 6.28$ & $V_{2}$ & $V_{1} \& V_{3}$ \\
$(2, \pm 2 \pi, 0)$ & $2 \sqrt{1+\pi^{2}} \sim 6.59$ & - & $V_{3}$ \\
$(0,3 \pi, 0)$ & $3 \pi \sim 9.42$ & $V_{2}$ & $V_{1} \& V_{3}$ \\
$(-2,3 \pi, 0)$ & $\sqrt{4+9 \pi^{2}} \sim 9.63$ & - & $V_{3}$ \\
& & & \\
\hline
\end{tabular}

Table 2: A list of the momenta studied showing which components of the vector current, $V_{i}=\bar{\psi} \gamma_{i} \psi$, are longitudinal or transverse. The momenta components $\vec{p} L$ which are multiples of $\pi$ are obtained from the usual Fourier sum, and those $p L$ components which are integer-valued are obtained from twisted boundary conditions.

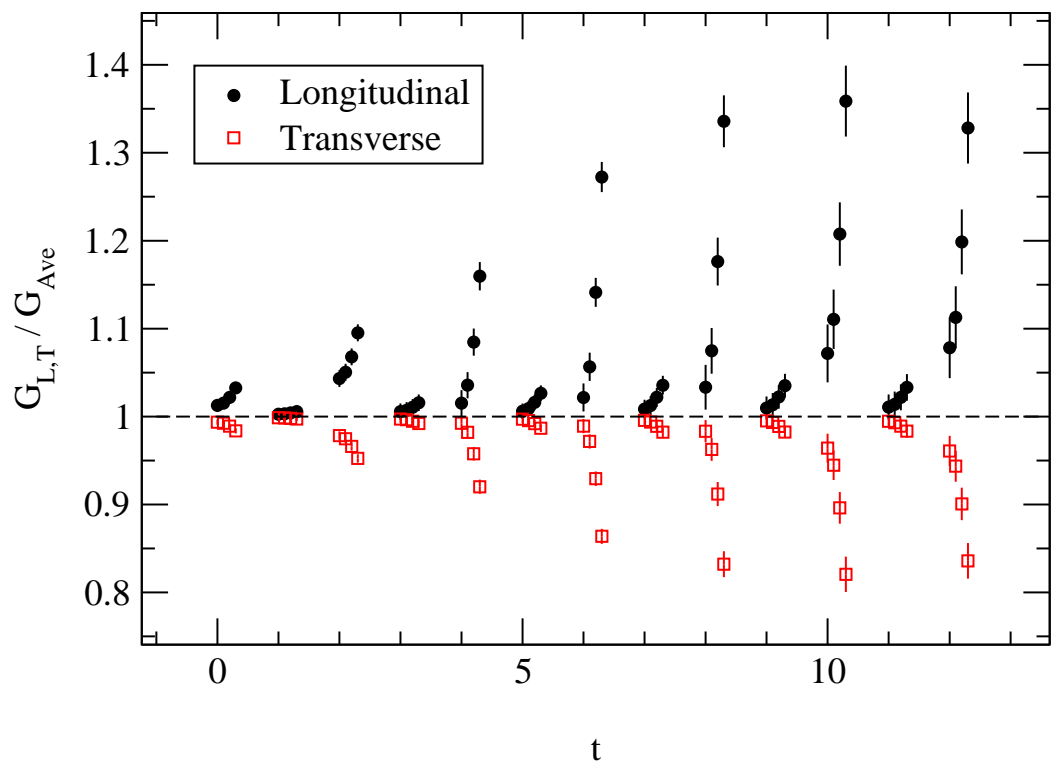

Figure 1: Longitudinal and transverse vector correlation functions normalised by the average correlation function, $G_{\mathrm{Ave}}=\left(G_{\mathrm{L}}+2 G_{\mathrm{T}}\right) / 3$, for various momenta, $\vec{p}$, as a function of euclidean time. Data points for each momentum (for a given time) are offset horizontally for clarity; from left to right they are $|\vec{p}| L=2, \pi$, $2 \pi$ and $3 \pi$. 

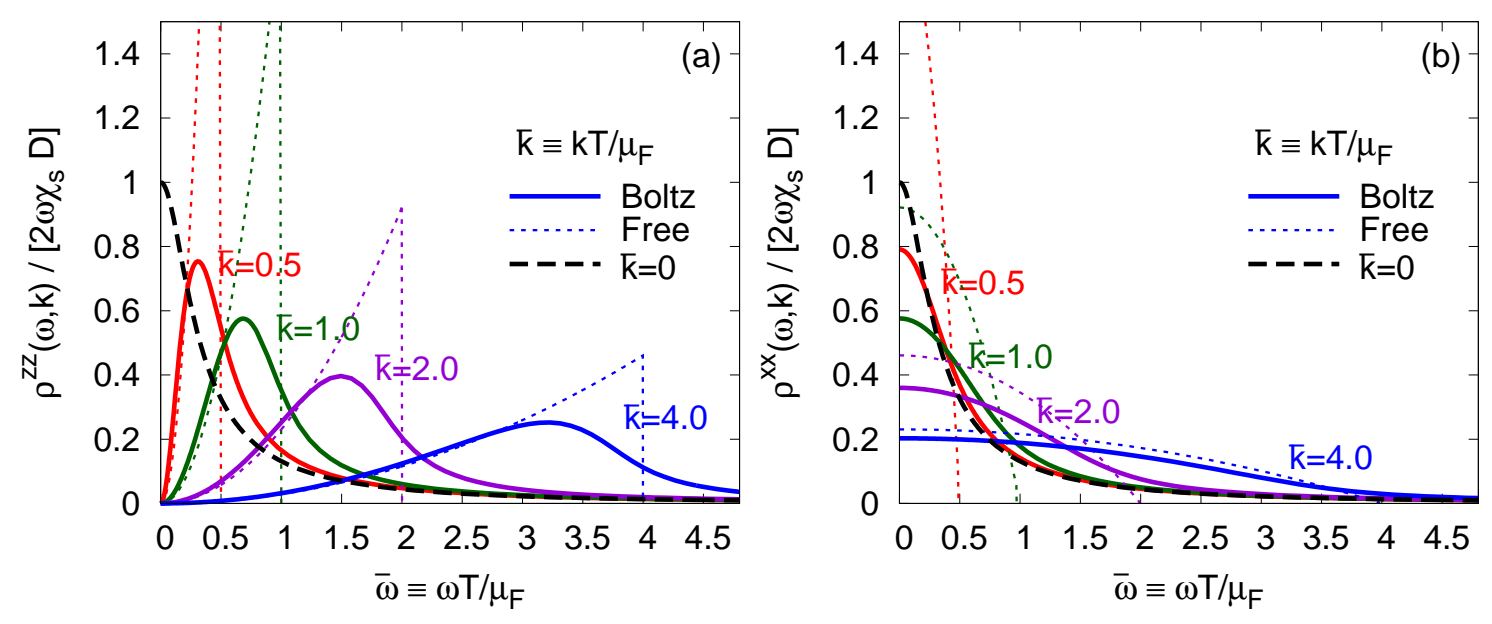

Figure 2: Longitudinal (left) and transverse (right) vector spectral function predictions from Ref. [4].
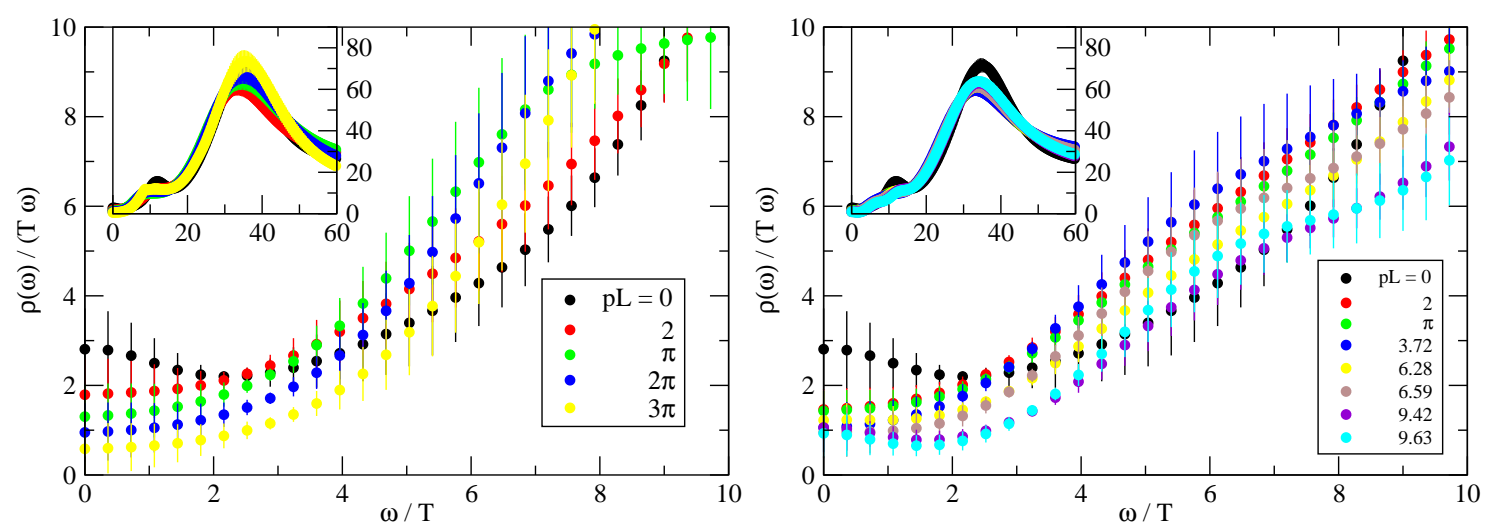

Figure 3: Longitudinal (left) and transverse (right) vector spectral function for the light ( $m a=0.01)$, hot case. The main plot shows the $\omega \sim 0$ region, and the insert the full $\omega$ range.

in our correlators definitions. As can be seen, there is a clear difference between these modes; the longitudinal correlator is consistently larger than the transverse for each time slice and momentum value. It would be interesting to understand this feature analytically. Note that the momentum dependence is much stronger for even timeslices. This is an artefact of the staggered formulation.

The diffusivity, $D$, can in principle be obtained from the momentum dependence of the vector spectral function (at small mass). In Ref. [4] a prediction was made of the energy dependence of the vector spectral function for both the longitudinal and transverse modes (for the massless case in the plasma phase). This is shown in Fig. 2. As can be seen, their prediction for the $\omega \rightarrow 0$ behaviour differs between these two modes: $\rho_{\mathrm{L}}(\omega=0, \vec{p})=0$ for $\vec{p} \neq 0$ whereas $\rho_{\mathrm{T}}(\omega=0, \vec{p}) \neq 0$.

In Fig. 3, we show the spectral functions obtained via MEM for the longitudinal and transverse vector case for quark mass $m a=0.01$. As can be seen, there is a clear non-zero intercept for both the longitudinal (contradicting [4]) and transverse modes. The spectral function for the heavier quark mass, $m a=0.05$, is shown in Fig. 4. We note that the intercept is zero for both the longitudinal and transverse cases for this heavier quark mass yielding a null result for the heavy-quark diffusion coefficient [14]. 

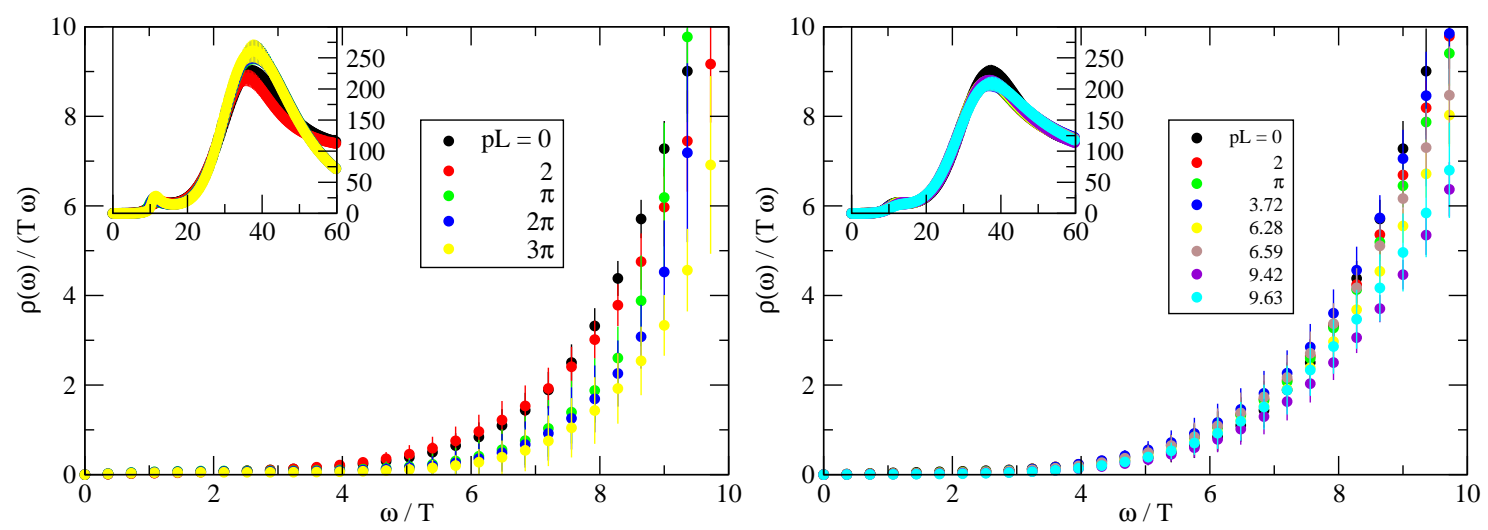

Figure 4: Longitudinal (left) and transverse (right) vector spectral function for the heavy, hot case. The main plot shows the $\omega \sim 0$ region, and the insert the full $\omega$ range.
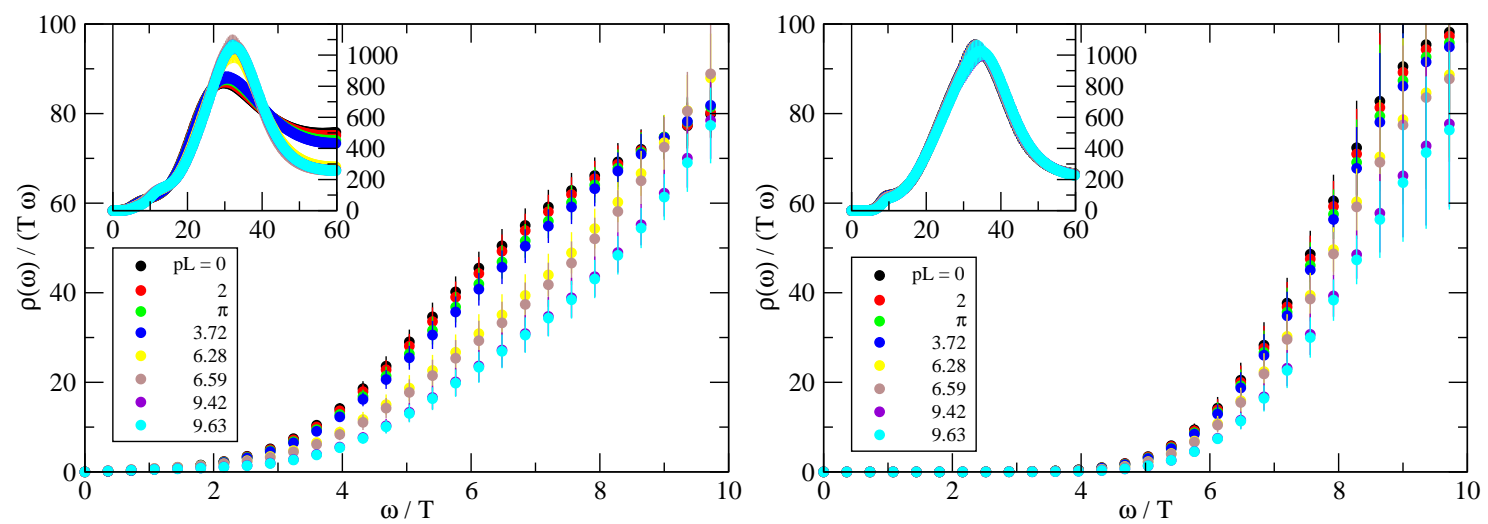

Figure 5: Pseudoscalar spectral function for the $m a=0.01$ (left) and $m a=0.05$ (right) cases. The main plot shows the $\omega \sim 0$ region, and the insert the full $\omega$ range.

We checked the spectral function for both the quark masses $m a=0.01$ and 0.05 in the cold phase, and found a zero intercept for both longitudinal and transverse modes. This agrees with our expectation that there are no transport features in this phase.

In Fig. 5, the pseudoscalar spectral functions are shown in the hot phase. We observe a zero intercept for this channel again in agreement with the expectation that there are no transport features.

\section{Conclusions}

In this work we have extended our previous study [2] of the spectral functions of vector correlators in the cold and hot phase of QCD to non-zero momenta using twisted boundary conditions to allow a finer momentum resolution. We are thus able to calculate and contrast the longitudinal and transverse modes of the vector two-point function. We find that a zero intercept at zero energy for the spectral function in all cases except for the hot, light quark vector mesons where we find $\rho(\omega \rightarrow 0, \vec{p}) \neq 0$ for both the longitudinal and transverse modes. This is despite the fact that the two 
modes' two-point correlation functions have a different qualitative behaviour. Our future plans are to study the momentum dependence of these modes with the aim of further studying hydrodynamic structure at zero and nonzero momentum [13].

\section{References}

[1] C. Amsler et al. [Particle Data Group], Phys. Lett. B 667, 1 (2008).

[2] G. Aarts, C. Allton, J. Foley, S. Hands and S. Kim, Phys. Rev. Lett. 99 (2007) 022002 [arXiv:hep-lat/0703008].

[3] G. Aarts, C. Allton, J. Foley, S. Hands and S. Kim, Nucl. Phys. A 785 (2007) 202 [arXiv:hep-lat/0607012]; PoS LAT2006 (2006) 134 [arXiv:hep-lat/0610061].

[4] See e.g. J. Hong and D. Teaney, arXiv:1003.0699 [nuvcl-th], and references therein.

[5] G. Aarts, PoS LAT2007 (2007) 001 [arXiv:0710.0739 [hep-lat]].

[6] H. B. Meyer, PoS LATTICE2008 (2008) 017 [arXiv:0809.5202 [hep-lat]].

[7] M. Asakawa, T. Hatsuda and Y. Nakahara, Prog. Part. Nucl. Phys. 46, 459 (2001) [arXiv:hep-lat/0011040].

[8] G. Aarts and J. M. Martinez Resco, JHEP 0204 (2002) 053 [arXiv:hep-ph/0203177].

[9] G. Aarts and J. M. Martinez Resco, Nucl. Phys. B 726 (2005) 93 [arXiv:hep-lat/0507004].

[10] S. Datta, F. Karsch, P. Petreczky and I. Wetzorke, Phys. Rev. D 69 (2004) 094507 [arXiv:hep-lat/0312037].

[11] F. Karsch, these proceedings.

[12] J. M. Flynn, A. Juttner and C. T. Sachrajda [UKQCD Collaboration], Phys. Lett. B 632 (2006) 313 [arXiv:hep-lat/0506016].

[13] G. Aarts, C. Allton, J. Foley, S. Hands and S. Kim, in preparation.

[14] P. Petreczky and D. Teaney, Phys. Rev. D 73 (2006) 014508 [arXiv:hep-ph/0507318]. 